\title{
GENERALISING QUASINORMAL SUBGROUPS
}

\author{
STEWART STONEHEWER
}

\author{
(Received 16 September 2011)
}

Dedicated to John Cossey on the occasion of his seventieth birthday

\begin{abstract}
In Cossey and Stonehewer ['On the rarity of quasinormal subgroups', Rend. Semin. Mat. Univ. Padova 125 (2011), 81-105] it is shown that for any odd prime $p$ and integer $n \geq 3$, there is a finite $p$-group $G$ of exponent $p^{n}$ containing a quasinormal subgroup $H$ of exponent $p^{n-1}$ such that the nontrivial quasinormal subgroups of $G$ lying in $H$ can have exponent only $p, p^{n-1}$ or, when $n \geq 4, p^{n-2}$. Thus large sections of these groups are devoid of quasinormal subgroups. The authors ask in that paper if there is a nontrivial subgroup-theoretic property $\mathfrak{X}$ of finite $p$-groups such that (i) $\mathfrak{X}$ is invariant under subgroup lattice isomorphisms and (ii) every chain of $\mathfrak{X}$-subgroups of a finite $p$-group can be refined to a composition series of $\mathfrak{X}$-subgroups. Failing this, can such a chain always be refined to a series of $\mathfrak{X}$-subgroups in which the intervals between adjacent terms are restricted in some significant way? The present work embarks upon this quest.
\end{abstract}

2010 Mathematics subject classification: primary 20E07; secondary 20E15.

Keywords and phrases: generalising quasinormality in finite $p$-groups.

\section{Introduction}

A subgroup $Q$ of a group $G$ is said to be quasinormal (sometimes permutable) if $\langle Q, H\rangle=Q H$ for all subgroups $H$ of $G$; that is, if $Q H=H Q$. We write $Q$ qn $G$. The concept is due to Ore [3], and in [4] he proved that, in finite groups, quasinormal subgroups are always subnormal. He also proved that they are modular. Recall that a subgroup $M$ of a group $G$ is modular if

$$
\langle X, M\rangle \cap Y=\langle X, M \cap Y\rangle \text { for all } X \leq Y
$$

and

$$
\langle X, M\rangle \cap Y=\langle X \cap Y, M\rangle \text { for all } X \text { and } Y \text { with } M \leq Y .
$$

In fact a subgroup $M$ of a finite group $G$ is quasinormal if and only if $M$ is modular and subnormal in $G$ (see [6, Theorem 5.1.1]). So in finite $p$-groups,

quasinormal subgroups and modular subgroups coincide.

(C) 2012 Australian Mathematical Publishing Association Inc. 0004-9727/2012 \$16.00 
Also they are clearly invariant under subgroup lattice isomorphisms (that is, projectivities).

It was explained in [1] why $p$-groups are so important in the theory of quasinormal subgroups of finite groups. For, suppose that $G$ is finite with $Q$ qn $G$ and $Q$ core-free. Then $Q$ is nilpotent and each Sylow $p$-subgroup $P$ of $Q$ is quasinormal in $G$. Also if $S$ is a Sylow $p$-subgroup $G$, then $P$ qn $S$ and the complexities of the embedding of $Q$ in $G$ are reduced to those of $P$ in $S$. Therefore in what follows we shall be mainly interested in finite $p$-groups. Our aim is to weaken the definition of quasinormality, while retaining its invariance under projectivities in finite $p$-groups, so as to produce a class of subgroups that are more involved with the structure of the group. We are particularly interested in relating group and lattice properties. An obvious starting point is to adopt just one of the definitions (1.1) and (1.2) of modularity.

Definition 1.1. We say that a subgroup $M$ of a group $G$ is semimodular if (1.1) holds (see [6, Exercise 7, p. 49]). Write $M \operatorname{sm} G$.

We should point out (and indeed the referee drew attention to the fact) that semimodularity of lattices is a standard concept, namely $X \cap Y$ maximal in $X$ implies $Y$ maximal in $\langle X, Y\rangle$ for all $X$ and $Y$ (see, for example, [7]). However, since we are concerned with individual elements rather than whole lattices, we have taken the liberty (following Schmidt in [6]) of using this terminology, which, while different from the original, will hopefully seem appropriate in the context. (The same applies in Definitions 1.4, 2.1 and 2.2 below.)

Given subgroups $H \leq K$ of a group, denote the lattice of subgroups between $H$ and $K$ by $[K / H]$. Then for subgroups $X, Y$ of a group $G$, define a map

$$
\Phi_{X, Y}:[X / X \cap Y] \rightarrow[\langle X, Y\rangle / Y]
$$

by

$$
H \mapsto\langle H, Y\rangle .
$$

Similarly, define a map

$$
\Psi_{X, Y}:[\langle X, Y\rangle / Y] \rightarrow[X / X \cap Y]
$$

by

$$
K \mapsto X \cap K
$$

In relation to semimodularity we have the following proposition.

Proposition 1.2. Let $M$ be a subgroup of $G$. Then:

(i) $M \operatorname{sm} G$ if and only if, for all $X \leq G$,

$$
\Phi_{X, M} \Psi_{X, M}=\operatorname{id}_{[X / X \cap M]}
$$

(ii) if $M \operatorname{sm} G$, then, for all $X \leq G$,

$$
\Psi_{M, X} \Phi_{M, X}=\operatorname{id}_{[\langle X, M\rangle / X]} .
$$


Both of these results are left to the reader in [6, Exercise 7, p. 49], and we do the same. However, when $G$ is a finite $p$-group there is a striking corollary.

Proposition 1.3. A semimodular subgroup of a finite p-group is quasinormal and therefore modular.

Proof. Let $G$ be a finite $p$-group with $M \operatorname{sm} G$ and let $X \leq G$. Then it follows from Proposition 1.2 that the map

$$
\Psi_{M, X}:[\langle X, M\rangle / X] \rightarrow[M / X \cap M]
$$

is injective. Therefore $|\langle X, M\rangle: X| \leq|M: X \cap M|$. However,

$$
|M: X \cap M|=|M| /|X \cap M|=|X M| /|X|,
$$

that is, $|\langle X, M\rangle| \leq|X M|$. Hence $\langle X, M\rangle=X M$ and so $M$ qn $G$.

So (1.2) is a redundant requirement in the definition of modular subgroups in finite $p$-groups. But of course we cannot remove the hypothesis that $G$ is a finite $p$-group in Proposition 1.3. For example, the subgroups of order two in the alternating group $A_{4}$ are semimodular, but not modular. As we stated above, however, our interest is in p-groups.

We consider next the defining property (1.2).

Definition 1.4. We say that a subgroup $M$ of a group $G$ is weakly modular if (1.2) holds. Write $M$ wm $G$.

Corresponding to Proposition 1.2(i) we have the following proposition.

Proposition 1.5. Let $M \leq G$. Then $M \mathrm{wm} G$ if and only if, for all $X \leq G$,

$$
\Psi_{X, M} \Phi_{X, M}=\operatorname{id}_{[\langle X, M\rangle / M]} .
$$

Proof. Suppose that $M$ wm $G, X \leq G$ and $M \leq Y \leq\langle X, M\rangle$. Then $Y=\langle X, M\rangle \cap Y=$ $\langle X \cap Y, M\rangle$, by (1.2). Therefore $\Psi_{X, M}: Y \mapsto X \cap Y$ and $\Phi_{X, M}: X \cap Y \mapsto\langle X \cap Y, M\rangle=Y$. Hence we have the necessary requirement.

Conversely, suppose that (1.3) holds and let $M \leq Y \leq G$. Then $X \cap Y=X \cap$ $(\langle X, M\rangle \cap Y)$. Therefore

$$
\Psi_{X, M}:\langle X, M\rangle \cap Y \mapsto X \cap Y \quad \text { and } \quad \Phi_{X, M}: X \cap Y \mapsto\langle X \cap Y, M\rangle .
$$

Thus, by (1.3), $\langle X, M\rangle \cap Y=\langle X \cap Y, M\rangle$ and so $M$ wm $G$.

Again we have a striking corollary.

Proposition 1.6. A weakly modular subgroup of a finite p-group is quasinormal and therefore modular. 
Proof. Let $G$ be a finite $p$-group with $M$ wm $G$ and let $X$ be a cyclic subgroup of $G$. It is sufficient to show that $\langle X, M\rangle=X M$. By Proposition $1.5, \Phi_{X, M}$ is a surjective map. Therefore since $[X / X \cap M]$ is a chain, it follows that $[\langle X, M\rangle / M]$ is a chain and

$$
|\langle X, M\rangle: M| \leq|X: X \cap M| .
$$

Thus $|X M|=|X||M| /|X \cap M|$ implies that $|\langle X, M\rangle: M| \leq|X M| /|M|$ and hence $|\langle X, M\rangle| \leq$ $|X M|$. Then $\langle X, M\rangle=X M$, as claimed.

It follows that either of the two conditions (1.1) and (1.2) for modularity is sufficient in finite p-groups. However, as before, we cannot remove the hypothesis that $G$ is a finite $p$-group in Proposition 1.6. For example, a maximal subgroup of any group is weakly modular.

So two possible lattice-invariant generalisations of the concept of quasinormality in finite $p$-groups have failed. In the next section we consider a third which at least produces a larger class of subgroups.

\section{Upper semimodular subgroups}

In [6, pp. 46, 47], Schmidt defines two generalisations of the concept of modular lattice, namely upper semimodular and lower semimodular. Applied to individual subgroups these are as follows. Let $M$ be a subgroup of a group $G$.

Definition 2.1. We say that $M$ is upper semimodular in $G$ (and we write $M$ usm $G$ ) if, for all $X \leq G$,

$$
X \cap M \text { maximal in } M \text { implies } X \text { maximal in }\langle X, M\rangle \text {. }
$$

Definition 2.2. We say that $M$ is lower semimodular in $G$ if, for all $X \leq G$,

$$
X \text { maximal in }\langle X, M\rangle \text { implies } X \cap M \text { maximal in } M \text {. }
$$

It is easy to see that in all finite p-groups, every subgroup is lower semimodular. However, an upper semimodular subgroup of a finite $p$-group is not always modular, as we see in the following example.

ExAmple. Let $p$ be an odd prime and let $G$ be the group generated by elements $a_{1}, a_{2}$, $a_{3}, b$ of order $p$ such that

$$
\left[a_{i}, b\right]=a_{i+1}, i=1,2 \quad \text { and } \quad\left[a_{3}, b\right]=\left[a_{i}, a_{j}\right]=1 \text {, all } i, j .
$$

Let $M=\left\langle a_{1}, a_{2}\right\rangle$. We claim that

$$
M \operatorname{usm} G .
$$

For, let $X \leq G$ with $|X \cap M|=p$ and $X \nless M$. We show that $|\langle X, M\rangle: X|=p$. Clearly we may assume that $X \nless A=\left\langle a_{1}, a_{2}, a_{3}\right\rangle$. Therefore there is an element $a b \in X$ with $a \in A$. We distinguish two cases. 
(i) Suppose that $X \cap M=\left\langle a_{2}\right\rangle$. Then $a_{3} \in X$ and so $|X| \geq p^{3}$. Hence $|X|=p^{3}$ and $G=X M$, that is, $X$ is maximal in $G$.

(ii) Suppose that $X \cap M \neq\left\langle a_{2}\right\rangle$. Then there is an element $a_{1} a_{2}^{i} \in X$, some $i$. Thus $a_{2} a_{3}^{i} \in X$ and $a_{3} \in X$. So $a_{2} \in X$, a contradiction.

Hence (2.2) follows. However, $M$ is not modular in $G$, since it is not quasinormal, that is, $M\langle b\rangle$ is not a subgroup.

Of course the property of being an upper semimodular subgroup is invariant under projectivities, that is,

upper semimodularity is a lattice-invariant property.

These subgroups also satisfy another important property, as we now show. We shall refer to them as usm subgroups.

Proposition 2.3. The join of two usm subgroups of a finite p-group is a usm subgroup.

Proof. Let $G$ be a finite $p$-group and $M_{1}, M_{2}$ usm $G$. Let $M=\left\langle M_{1}, M_{2}\right\rangle$ and suppose that $X \leq G$ with $X \cap M$ maximal in $M$. We write $X \cap M \lessdot M$. So $|M: X \cap M|=p$. We show that

$$
X \lessdot\langle X, M\rangle \text {. }
$$

Clearly $M \nless X$ and so without loss of generality we may assume that $M_{1} \nless X$. But $\left\langle X, M_{1}\right\rangle=\left\langle X, X \cap M, M_{1}\right\rangle=\langle X, M\rangle$ and $X \lessdot\left\langle X, M_{1}\right\rangle$ since $X \cap M_{1} \lessdot M_{1}$. So (2.3) follows.

We saw in the above example that usm subgroups of finite $p$-groups are not quasinormal in general. In that example the subgroup is elementary abelian of rank two. It could not have had order $p$ for the following reason.

Proposition 2.4. A usm subgroup of order $p$ in a finite p-group is quasinormal.

Proof. Let $G$ be a finite $p$-group with $M$ usm $G$ and $|M|=p$. Let $X \leq G$. Then

$$
\langle X, M\rangle=X M
$$

For we may assume that $M \nless X$. Thus $M \cap X=1$ and so by hypothesis $|\langle X, M\rangle: X|=p$. Hence $X \triangleleft\langle X, M\rangle$ and (2.4) follows.

In fact the above result is true even for cyclic usm subgroups. But in order to prove this we need two elementary properties.

Proposition 2.5. Let $M$ be a usm subgroup of a group $G$.

(i) If $M \leq H \leq G$, then $M$ usm $H$.

(ii) If $\theta$ is a homomorphism of $G$, then $M \theta$ usm $G \theta$. 
Proof. (i) This is clear.

(ii) Let $N \triangleleft G$. Then it suffices to show that $M N / N$ usm $G / N$. Let $X / N \leq G / N$ such that $X \cap M N \lessdot M N$. We have

$$
M N / N \cong M /(N \cap M)
$$

and under this isomorphism $(X \cap M) N / N=(X \cap M N) / N$ corresponds to $(X \cap M) /$ $(N \cap M)$. Therefore $X \cap M \lessdot M$ and so $X \lessdot\langle X, M\rangle$, by hypothesis, that is, $X / N \lessdot$ $\langle X / N, M N / N\rangle$. Hence $M N / N$ usm $G / N$.

Now we can show that Proposition 2.4 is just the simplest case of a more general result. We denote the subgroup generated by the elements of order $p$ in a $p$-group $G$ by $\Omega_{1}(G)$.

THEOREM 2.6. A cyclic usm subgroup of a finite p-group is quasinormal.

Proof. Let $G$ be a finite $p$-group with $M$ usm $G$ and $M$ cyclic. Suppose for a contradiction that $M$ is not quasinormal in $G$ and let $G$ be a counterexample of minimal order. Then there is a cyclic subgroup $X$ of $G$ such that $X M \neq M X$. Also $G=\langle X, M\rangle$, by Proposition 2.5(i) and choice of $G$.

Let $N$ be a minimal normal subgroup of $G$. Then again by choice of $G$ and Proposition 2.5(ii), $G=N X M$. We claim that

$$
N=Z_{1}(G)
$$

the centre of $G$. For otherwise there exists an element $x y \in Z_{1}(G), x y \neq 1, x \in X$, $y \in M$. Then $[x, y]=1$ and so $\langle x y\rangle \subset X M$. But $G=\langle x y\rangle X M$, since $G /\langle x y\rangle$ is not a counterexample. Therefore $G=X\langle x y\rangle M=X M$, contradiction. Thus (2.5) is true.

By [6, Theorem 5.2.13], $G / N$ is metacyclic, being the product of two cyclic groups. Therefore there are cyclic subgroups $H, K$ of $G$ such that

$$
N H \triangleleft G=N H K .
$$

Let $H=\langle h\rangle, K=\langle k\rangle$. So we may assume that

$$
h^{k}=u h^{1+\lambda p},
$$

where $u \in N, \lambda$ an integer. Suppose that $|H|=p$. If also $|K|=p$, then $|G|=p^{3}$. If $p$ is odd, $G$ must have exponent $p$. But then $|M|=p$, contradicting Proposition 2.4. On the other hand, if $p=2$, then $G \cong D_{8}$, the dihedral group of order eight, with $M$ of order four, again by Proposition 2.4. So $M \triangleleft G$, a contradiction. Thus we must have $|K| \geq p^{2}$. Then $K^{p} \leq Z_{1}(G)$, by (2.6), and so $K^{p}=N$, by (2.5). Therefore again $|G|=p^{3}$ and we easily obtain contradictions. Thus

$$
|H| \geq p^{2} .
$$

It follows from (2.6) that $\Omega_{1}(H) \leq Z_{1}(G)$ and so $\Omega_{1}(H)=N$ and $G=H K$, with $H \triangleleft G$ and $H$ and $G / H$ cyclic. If $p$ were odd, then all its subgroups would be 
quasinormal, by [6, Theorem 2.3.1]. So $p=2$. We shall see in the next lemma that $M$, not being quasinormal in $G$, cannot lie in the subgroup of $G$ of index two which contains $H$. But then we have $G=H M$. Also $G / H^{4}$ has a quotient isomorphic to $D_{8}$ in which the image of $M$ is noncentral of order two (again by [6, Theorem 2.3.1]). However, this contradicts Propositions 2.4 and 2.5(ii) and completes the proof of the theorem.

Lemma 2.7. Let $G$ be a finite 2-group with $H \triangleleft G, H$ cyclic of order at least four, and let $G / H$ be cyclic and nontrivial. Let $L / H$ be the subgroup of index two in $G / H$. Then every subgroup of $L$ is quasinormal in $G$.

Proof. Suppose that the lemma is false and let $G$ be a counterexample of minimal order. Let $M \leq L$ with $M$ not quasinormal in $G$. Then $H \cap M=1$, otherwise $M / H \cap M$ is quasinormal in $G / H \cap M$, by choice of $G$, and thus $M$ qn $G$, a contradiction. Therefore $M$ is cyclic. Let $X$ be a cyclic subgroup of $G$ such that

$$
\langle M, X\rangle \neq M X .
$$

Again by [6, Theorem 2.3.1], we see that $L$ is a modular group, that is, all its subgroups are modular, therefore quasinormal (in $L$ ). Thus $X \nless L$ and so $G=H X$. Also we must have $H \cap X=1$, otherwise $M X / H \cap X$ is a subgroup by choice of $G$.

Let $M=\langle h x\rangle, h \in H, x \in X^{2}$. Then $h \in M X$. Also $h \neq 1$. Now

$$
(h x)^{2}=h h^{1+4 \lambda} x^{2},
$$

for some integer $\lambda$. Squaring this equation repeatedly, we see that some power $h_{1} x_{1}$ $\left(h_{1} \in H, x_{1} \in X^{2}\right)$ of $h x$ has $\left|h_{1}\right|=2$. Thus $\left\langle h_{1}\right\rangle \in M X \cap Z_{1}(G)$ and again, by choice of $G, M X /\left\langle h_{1}\right\rangle$ is a subgroup of $G /\left\langle h_{1}\right\rangle$. This contradicts (2.7) and so the lemma follows.

The above results suggest that the concept of upper semimodularity might be a useful lattice-invariant generalisation of quasinormality in finite $p$-groups, with a view to understanding more about the connection between group and subgroup-lattice structures. But it turns out that there are two properties of quasinormal subgroups, one extremely useful and the other quite intriguing, neither of which is inherited by usm subgroups. The first concerns the intersection of a quasinormal subgroup $Q$ with an arbitrary subgroup $H$ in a group. The intersection $Q \cap H$ is always quasinormal in $H$. However, this property is not possessed by usm subgroups. To see this, let $G$ be the group of the previous example. Then $M=\left\langle a_{1}, a_{2}\right\rangle$ usm $G$. Let $H=\left\langle a_{2}, a_{3}, b\right\rangle$, so $H \cap M=\left\langle a_{2}\right\rangle$. Clearly $\left\langle a_{2}\right\rangle$ is not quasinormal in $H$. Therefore $\left\langle a_{2}\right\rangle$ is not a usm subgroup of $H$, by Proposition 2.4.

In fact the same example illustrates the second property of quasinormal subgroups referred to above. It was shown in [2] that for odd primes $p$, a quasinormal subgroup of order $p^{2}$ in a finite p-group $G$ always contains a quasinormal subgroup (of $G$ ) of order $p$. However, it is easy to see that in the example above, where $M$ has order $p^{2}$, 
there is no usm subgroup of $G$ of order $p$ lying in $M$. For, such a subgroup would have to be quasinormal in $G$, by Proposition 2.4. Thus upper semimodularity is not a candidate for the class $\mathfrak{X}$ referred to at the beginning of this paper.

There is yet a third property of quasinormal subgroups that is not inherited by usm subgroups. A subgroup $M$ that permutes with all cyclic subgroups is quasinormal. But again take $G$ in our example, with $p \geq 5$, and let $M=\left\langle a_{1}, a_{3}\right\rangle$. Since $G$ has exponent $p$, each nontrivial cyclic subgroup $X$ of $G$ with $X \cap M \lessdot M$ lies in $M$ and so (2.1) holds. But if $M$ usm $G$, then modulo $\left\langle a_{3}\right\rangle, M$ would be quasinormal in $G$ (by Propositions 2.4 and 2.5(ii)). Thus $M$ would be quasinormal in $G$, which is not the case.

\section{Joins as restricted products}

We conclude this preliminary investigation by considering another concept generalising quasinormality. By definition, $H$ qn $G$ if and only if $\langle H, K\rangle=H K$ for all $K \leq G$. Of course when $G$ is finite, for any two subgroups $H$ and $K$ of $G$, we always have

$$
\langle H, K\rangle=(H K)^{n}
$$

for some finite $n$. (Here for sets $X, Y, X Y=\{x y \mid x \in X, y \in Y\}$.) In this connection there is an interesting elementary result.

Proposition 3.1. Let $G$ be a finite p-group with subgroups $H, K$ such that $G=H K H$. Then $G=H K$.

Proof. We proceed by induction and assume the result is true for groups of order less than $|G|$. Let $h_{1} k h_{2}$ be a central element of order $p$, with $h_{1}, h_{2} \in H, k \in K$. Thus $h_{1} k h_{2}$ commutes with $k h_{2}$ and so $h_{1}$ commutes with $k h_{2}$. Therefore $h_{1} k h_{2}=k h_{2} h_{1}$. Similarly, $h_{2}$ commutes with $h_{1} k$. Put $h=h_{2} h_{1}$. Then

$$
h k=h_{2} h_{1} k=h_{1} k h_{2}=k h_{2} h_{1}=k h .
$$

Thus $N=\langle h k\rangle \subset H K$ and $N \leq Z_{1}(G)$. By induction,

$$
G / N=H K N / N=H N K / N=H K / N
$$

and hence $G=H K$ as required.

For our final generalisation of quasinormality, we shall use the fact that a subgroup is quasinormal if and only if its join with every cyclic subgroup is just its product.

Definition 3.2. We call a subgroup $H$ of a group $G$ 4-quasinormal, and write $H \mathrm{qn}_{4} G$, if

$$
\langle H, K\rangle=H K H K,
$$

for all cyclic subgroups $K$ of $G$. 
Clearly this concept can be generalised successively to $n$-quasinormal by increasing the number of factors $H, K$. In this way, eventually every subgroup $H$ of every finite group will be included.

We shall restrict ourselves to finite $p$-groups. Given the complexity of these groups, the difficulties of discovering the properties of even 4-quasinormal subgroups will surely be considerable. However, we have been able to make a start when $|H|=p$ by establishing their lattice-invariance.

Theorem 3.3. Let $p$ be a prime and let $G, \bar{G}$ be lattice-isomorphic finite p-groups. Suppose that $H \mathrm{qn}_{4} G$ and $|H|=p$. Denote the image of $H$ in $\bar{G}$ by $\bar{H}$. Then $\bar{H} \mathrm{qn}_{4} \bar{G}$.

Proof. We have to show that for every cyclic subgroup $\bar{K}$ of $\bar{G}$,

$$
\langle\bar{H}, \bar{K}\rangle=\bar{H} \bar{K} \bar{H} \bar{K} .
$$

Of course the pre-image $K$ of $\bar{K}$ is cyclic of the same order as $\bar{K}$, say $p^{n}, n \geq 1$. Thus we may assume that

$$
G=\langle H, K\rangle=H K H K
$$

and $\bar{G}=\langle\bar{H}, \bar{K}\rangle$ having the same order as $G$. Let $N=K_{G}$, the core of $K$ in $G$. Then (continuing to use bars to denote images) $\bar{N}$ qn $\bar{G}$ and so $\bar{H}$ normalises $\bar{N}$. Therefore $\bar{N} \triangleleft \bar{G}$ and clearly $\bar{N}=\bar{K}_{\bar{G}}$. Since 4-quasinormality is obviously preserved by homomorphisms, we may assume that

$$
K_{G}=1 \text { and } \bar{K}_{\bar{G}}=1 .
$$

From (3.1), we see that $|\bar{G}|=|G| \leq p^{2 n+1}$. Let $\bar{H}=\langle h\rangle, \bar{K}=\langle k\rangle$. Then

$$
\text { the double cosets } \bar{H} k^{i} h \bar{K}, 0 \leq i \leq p^{n}-1 \text {, are distinct. }
$$

For suppose that $\bar{H} k^{i} h \bar{K}=\bar{H} k^{j} h \bar{K}$ with $i \neq j, 0 \leq i, j \leq p^{n}-1$. Then

$$
k^{i} h=h_{1} k^{j} h k_{1} \text {, }
$$

where $h_{1} \in \bar{H}, k_{1} \in \bar{K}$. Therefore $k^{i}=h_{1} k^{j} k_{1}\left[k_{1}, h^{-1}\right]$. It follows from (3.4) that $k_{1} \neq 1$. So $\left[k_{1}, h^{-1}\right] \neq 1$, by (3.2), and hence $h_{1}^{-1} k^{i-j} k_{1}^{-1} \in \bar{G}^{\prime} \backslash\{1\}$. Since $\bar{G}=\langle h, k\rangle$, we must have $h_{1}=1$, otherwise $\bar{G}=\left\langle h_{1}^{-1} k^{i-j} k_{1}^{-1}, k\right\rangle=\langle k\rangle$, a contradiction. Thus, by (3.4),

$$
k_{1}^{h^{-1}} \in \bar{K} \text {. }
$$

But this contradicts (3.2). Therefore (3.3) follows.

Finally, for $g \in \bar{G}$,

$$
|\bar{H} g \bar{K}|=|\bar{H}||\bar{K}| /\left|\bar{H}^{g} \cap \bar{K}\right|=p^{n+1}
$$

(see [5, p. 90, Exercise 247]). Thus from (3.3) we see that $|\bar{H} \bar{K} \bar{H} \bar{K}| \geq p^{2 n+1}$. Therefore $\bar{G}=\bar{H} \bar{K} \bar{H} \bar{K}$.

An obvious question is whether Theorem 3.3 extends to arbitrary cyclic $\mathrm{qn}_{4}$ subgroups or indeed to all $\mathrm{qn}_{4}$-subgroups, and we have made some progress in this direction. Also, which other properties of quasinormal subgroups are inherited by, or can be generalised to, $\mathrm{qn}_{4}$-subgroups? We make no conjectures, but hopefully more results will appear in due course. 


\section{References}

[1] J. Cossey and S. E. Stonehewer, 'On the rarity of quasinormal subgroups', Rend. Semin. Mat. Univ. Padova 125 (2011), 81-105.

[2] J. Cossey, S. E. Stonehewer and G. Zacher, 'Quasinormal subgroups of order $p^{2}$, Ric. Mat. 57 (2008), 127-135.

[3] O. Ore, 'Structures and group theory I', Duke Math. J. 3 (1937), 149-173.

[4] O. Ore, 'On the application of structure theory to groups', Bull. Amer. Math. Soc. 44 (1938), 801-806.

[5] J. S. Rose, A Course on Group Theory (Cambridge University Press, Cambridge, 1978).

[6] R. Schmidt, Subgroup Lattices of Groups (de Gruyter, Berlin, 1994).

[7] M. Stern, Semimodular Lattices (Cambridge University Press, Cambridge, 1999).

STEWART STONEHEWER, Mathematics Institute, University of Warwick, Coventry CV4 7AL, UK

e-mail: S.E.Stonehewer@warwick.ac.uk 